\title{
Leaf Disease Classification using Advanced SVM Algorithm
}

\author{
Rima Herlina S. Siburian, Rahmi Karolina, Phong Thanh Nguyen, E. Laxmi Lydia, K. Shankar
}

\begin{abstract}
Presently there are many alternates of pesticides and unfortunately a very big portion of the industry is relies and using such poisons to protects crops to prevent from bugs attack and spreading of infection. Such pesticides are seriously very harmful and used unorganic chemicals. Even some of such pesticides are beneficial for insects too. Even some times there is also an possibility that such chemicals may be automatically washed during rain or watering the crops. So the research since years on green house agro system focus on early pest detection. Such methodology focus on observing plants by camera. The images captured by cameras can be used to analyzed that weather the plants are infected or not. $A$ number of methods and algorithms such as color conversion, segmentation, $k$-mean, knn etc are used to classified such images. This research is focusing on the interpretation of image for early stage pest detection so that the crop should be prevented from damage.
\end{abstract}

Keywords:- Early PEST Detection, Segmentation Algorithm, Pesticides alternates, Binary Image Conversion.

\section{INTRODUCTION}

This research primarily focuses on greenhouse crops. Image processing strategy assumes a significant job in the discovery of the irritations. Truly there are different trades of pesticides and incredibly a vital bit of the business is depends and utilizing such risky substances to shields harvests to keep from bugs trap and spreading of debasement. Such pesticides are actually fantastically risky and utilized inorganic produced substances. Beyond question, even some of such pesticides are beneficial for bugs too. In actuality, even a few times there is in like way an acceptability that such synthetics might be in this way washed during precipitation or watering the harvests. So the evaluation since years on green house agro framework base on early bug conspicuous confirmation. Such framework base on watching plants by camera. The photographs gotten by cameras can be utilized to investigated that climate the plants are undermined or not.

Revised Manuscript Received on July 22, 2019.

Rima Herlina S. Siburian, Universitas Papua, Indonesia.

Phong Thanh Nguyen, Department of Project Management, Ho Chi Minh City Open University, Vietnam.

E. Laxmi Lydia, Vignan's Institute of Information Technology (A), Department of Computer Science and Engineering, Visakhapatnam, Andhra Pradesh, India. India

K. Shankar, Department of Computer Applications Alagappa University,
Rahmi Karolina, Universitas Sumatera Utara, Indonesia.

Various methods and tallies, for example, hiding change, division, k-mean, knn, and so on are utilized to mentioned such pictures. This examination is concentrating on the translation of picture for beginning time inconvenience affirmation with the target that the yield ought to be kept from underhandedness. The principal goal is to distinguish some example bug. To accomplish this some advanced picture preparing calculations are gained. The model framework demonstrated dependable for fast location of bug. All techniques in this paper spare time and give proficient outcome. The strategies utilized are DBSCAN and NN calculations. The by and large early nuisance recognition exactness is $96 \%$ dependent on complete irritation dataset utilized in research.

Directly there are numerous interchanges of pesticides and shockingly a major part of the business is depends and utilizing such toxic substances to shields harvests to keep from bugs assault and spreading of contamination. Such pesticides are genuinely extremely destructive and utilized inorganic synthetic substances. Indeed, even some of such pesticides are valuable for bugs as well. Indeed, even a few times there is likewise a plausibility that such synthetics might be consequently washed during precipitation or watering the harvests. So the examination since years on green house agro framework center around early bug identification. Such strategy center around watching plants by camera. The pictures caught by cameras can be utilized to investigated that climate the plants are tainted or not. Various strategies and calculations, for example, shading transformation, division, kmean, knn and so forth are utilized to ordered such pictures. This exploration is concentrating on the translation of picture for beginning time bother recognition with the goal that the yield ought to be kept from harm.

\section{Background History}

Beneath gave is a short outline of the calculations that are at present utilized for vermin distinguishing proof by various researcher's exploration.

In paper [1] creators present picture preparing strategy for Rice malady distinguishing proof and considered the two most normal infections in the north east India, to be specific Leaf Blast (Magnaporthe Grisea) and Brown Spot (Cochio bolus Miyabeanus).

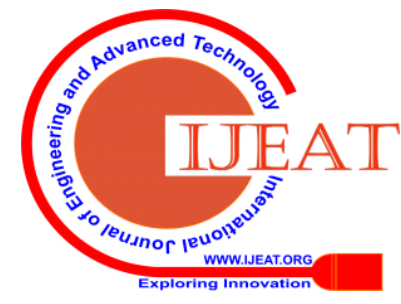




\section{Leaf Disease Classification using Advanced SVM Algorithm}

Picture procurement is fundamental advance, after that creator use division, limit discovery and spot identification strategy for highlight extraction of the contaminated pieces of the leave. In this paper creator presents zooming calculation in which SOM (Self Organizing Map) neural system is utilized for characterization sick rice pictures.

\section{STUDY}

\section{AIM}

The focus of this research is to detect pest from dataset images or camera images. Further on the basis of features extraction the type of pesticides can be decided for use.

\section{Existing Classification Algorithms}

\section{SVM Algorithm}

Support Vector Machines (SVMs) are a moderately new directed order procedure to the land spread mapping network. They have their foundations in Statistical Learning Theory and have picked up noticeable quality since they are hearty, precise and are compelling notwithstanding when utilizing a little preparing test.

\section{k-NN Classification}

In example acknowledgment, the k-closest neighbors calculation (k-NN) is a non-parametric strategy utilized for characterization and regression.[1] In the two cases, the info comprises of the $\mathrm{k}$ nearest preparing models in the component space. The yield relies upon whether $\mathrm{k}-\mathrm{NN}$ is utilized for order or relapse.

\section{k-means Clustering Algorithm}

k-implies grouping is a technique for vector quantization, initially from sign handling, that is prevalent for bunch investigation in information mining. k-implies grouping expects to parcel $\mathrm{n}$ perceptions into $\mathrm{k}$ bunches in which every perception has a place with the bunch with the closest mean, filling in as a model of the bunch. This outcomes in a dividing of the information space into Voronoi cells.

\section{OBJECTIVES}

The research focus on three main objectives given below:

1. Detection of different types of pest on the basis of features extraction

2. Suggesting the right pesticides

3. Minimize the manual efforts

\section{PROPOSED METHOdOLOGY}

\section{Problem Definition}

Truly there are different trades of pesticides and incredibly a vital bit of the business is depends and utilizing such risky substances to shields harvests to keep from bugs trap and spreading of debasement. Such pesticides are actually fantastically risky and utilized inorganic produced substances. Beyond question, even some of such pesticides are beneficial for bugs too. In actuality, even a few times there is in like way an acceptability that such synthetics might be in this way washed during precipitation or watering the harvests. So the evaluation since years on green house agro framework base on early bug conspicuous confirmation. Such framework base on watching plants by camera. The photographs gotten by cameras can be utilized to investigated that climate the plants are undermined or not. Various methods and tallies, for example, hiding change, division, k-mean, knn, and so on are utilized to mentioned such pictures.

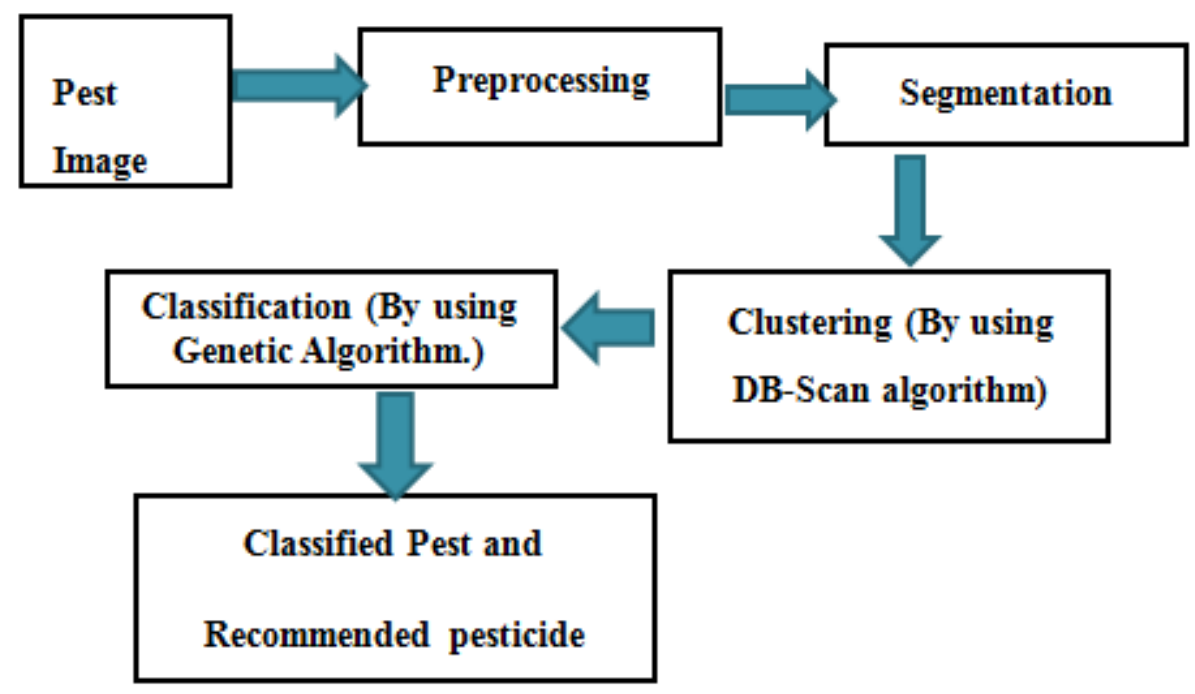

Fig. 1: System Architecture 
Clustering To Detect, Classify and Separate Plant and Pest

a. Green Pixels are masked and removed

Segmentation

b. Important datasets segmentations obtained

c. Final Clustering To Separate Pest Images Based On Image Subtraction

\subsubsection{Algorithmic Steps}

- Image Pre-processing

- Detection of Pests in the Image

- Filtering of the Image

There are two algorithm are used to develop the system :

- DB-SCAN

- Genetic Nural network

- Extraction of the Detected Pests

Flowchart :

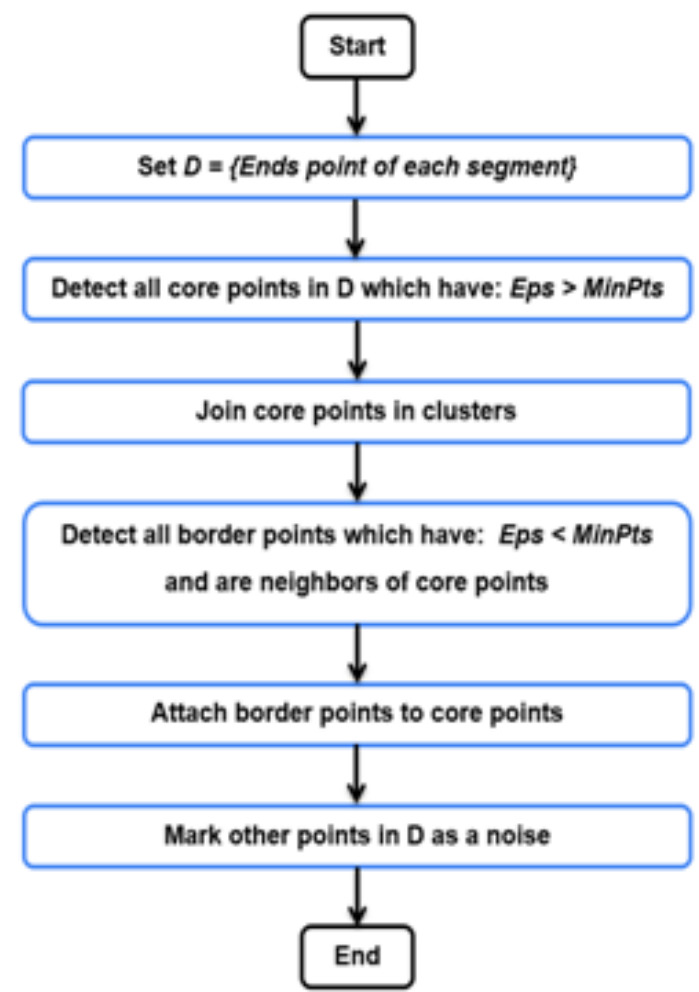

Fig. 2: Flowchart of DB -Scan

\section{IMPLEMENTATION}

MATLAB can be accessed either by start menu or by clicking on shortcut icon on the desktop. Once its IDE is launched its default layout will be appear. This window contains working area, programming area and its functions can be accessed by its menu.

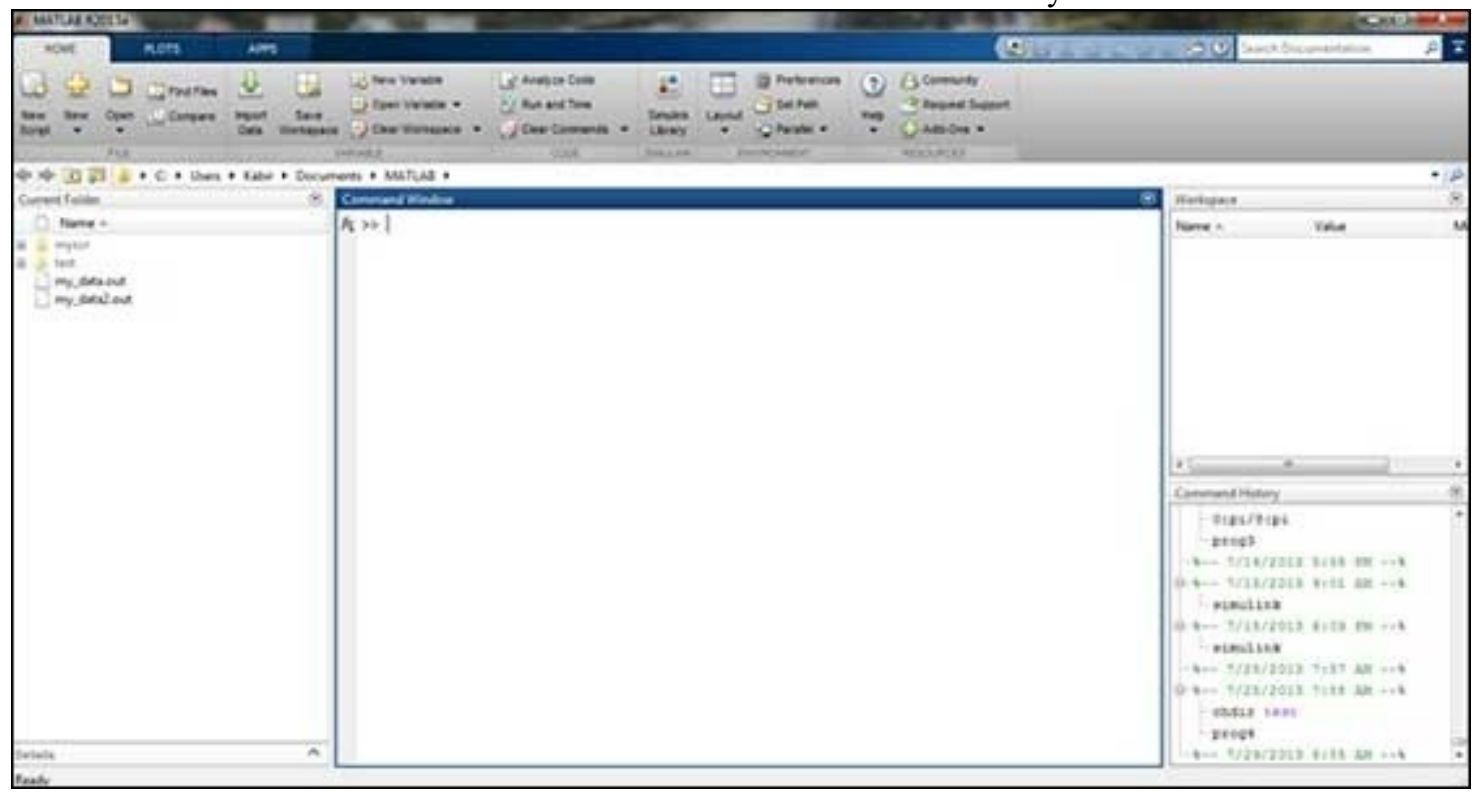

Fig 3: MATLAB environment 


\section{Leaf Disease Classification using Advanced SVM Algorithm}

\section{Implementation details :}

The implementation of this project is done using MATLAB .To open the project first open the MATLAB software .Set the particular path of source code and press on run

button

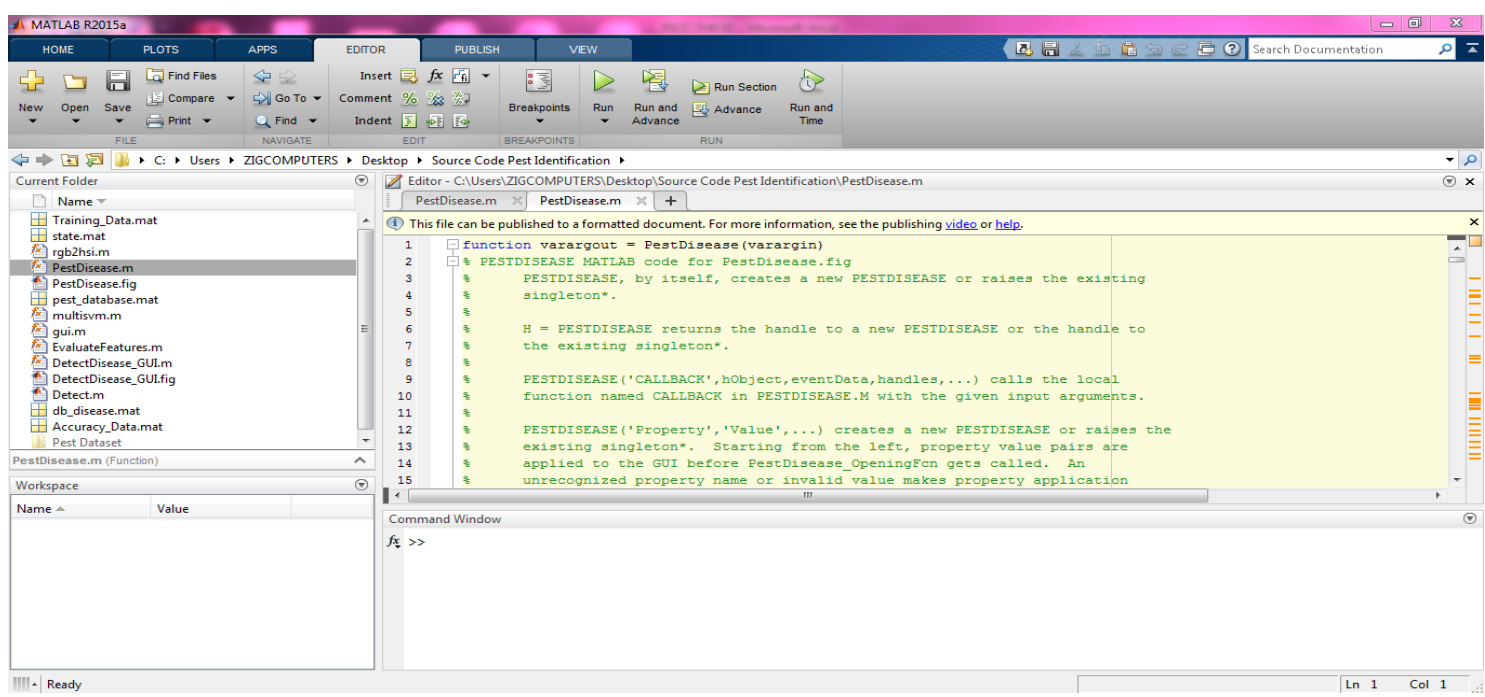

Fig 4 : MATLAB environment for project

After clicking on the run button, This project start running the source code in MATLAB. After running the code window will appear. The snapshot of this is as follow:

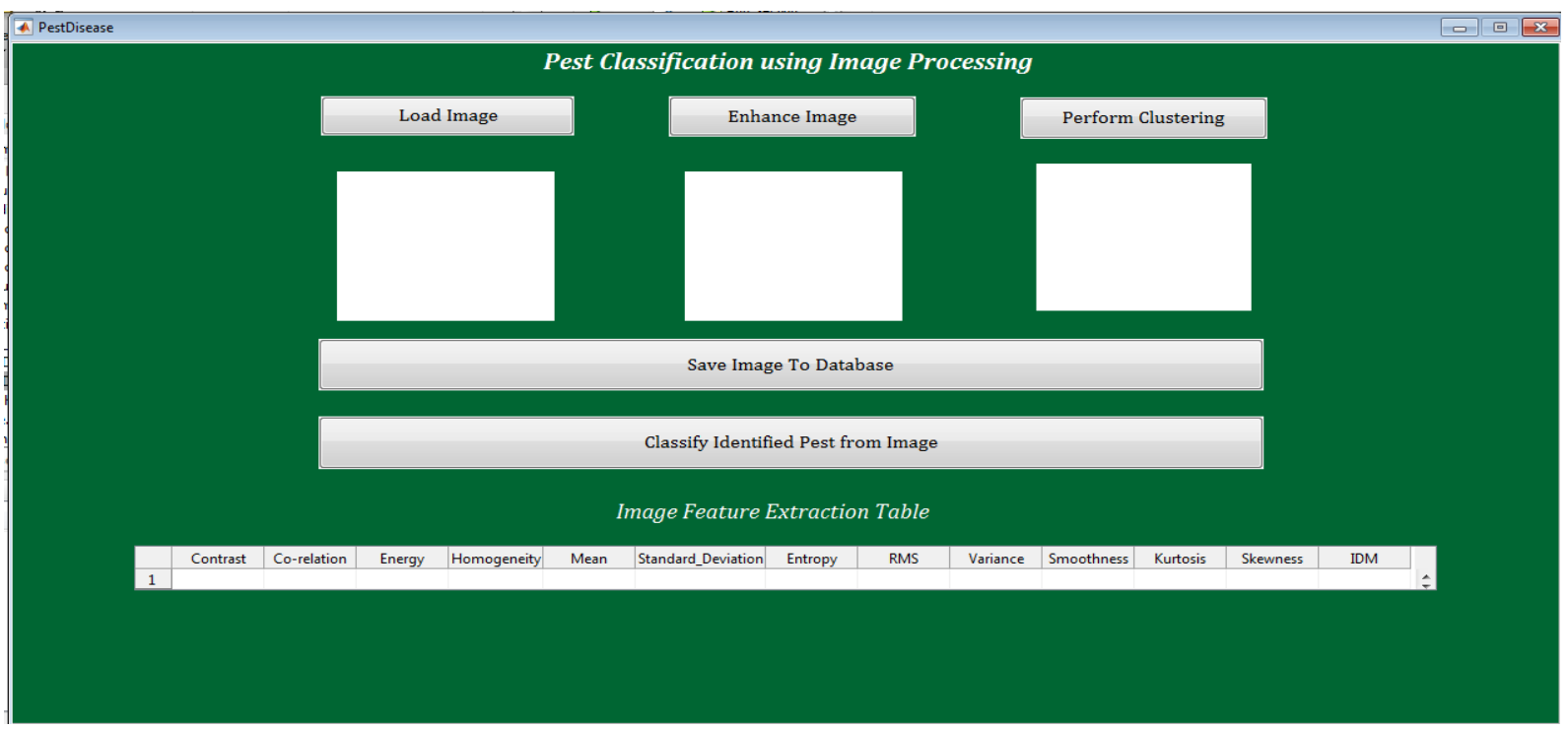

Fig 5 : Running the project in the MATLAB

\section{System execution details :}

If user click on the "Load Image" button then the data set of the pest images are open then select one of the image from the dataset.

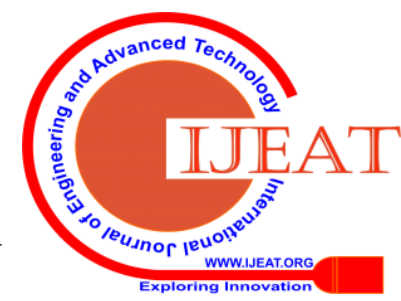




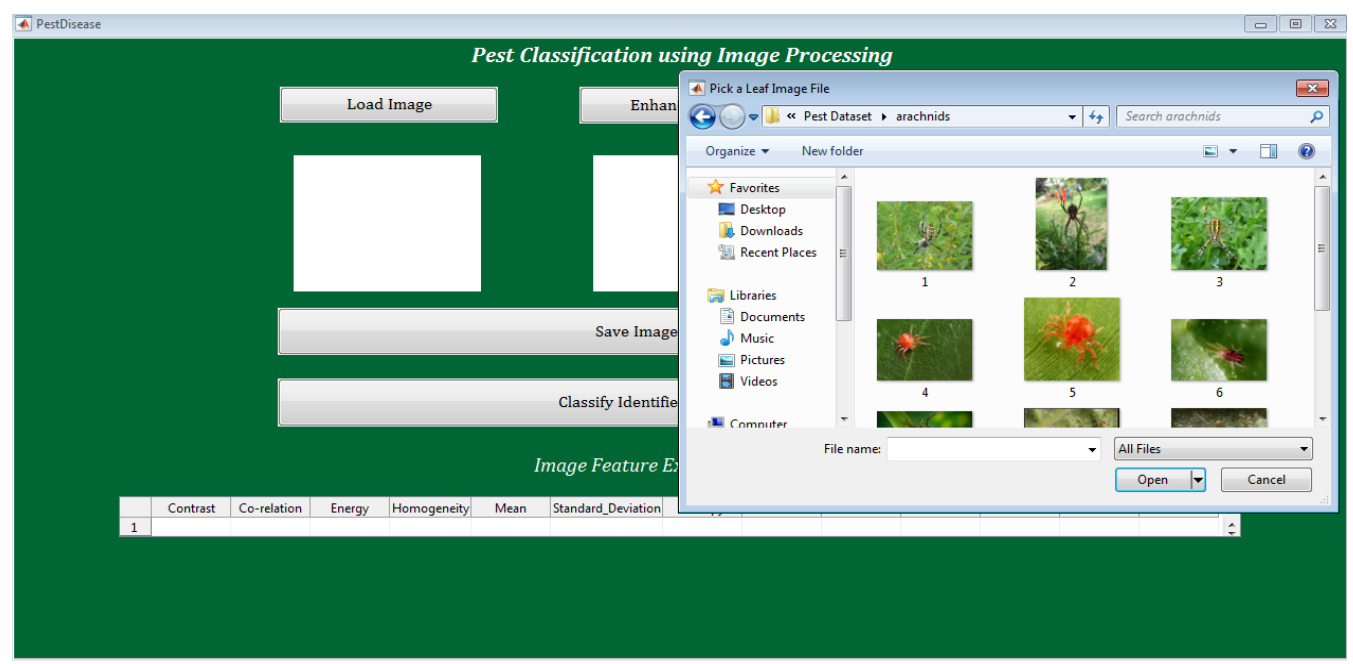

Fig 6: Load image as input from the dataset

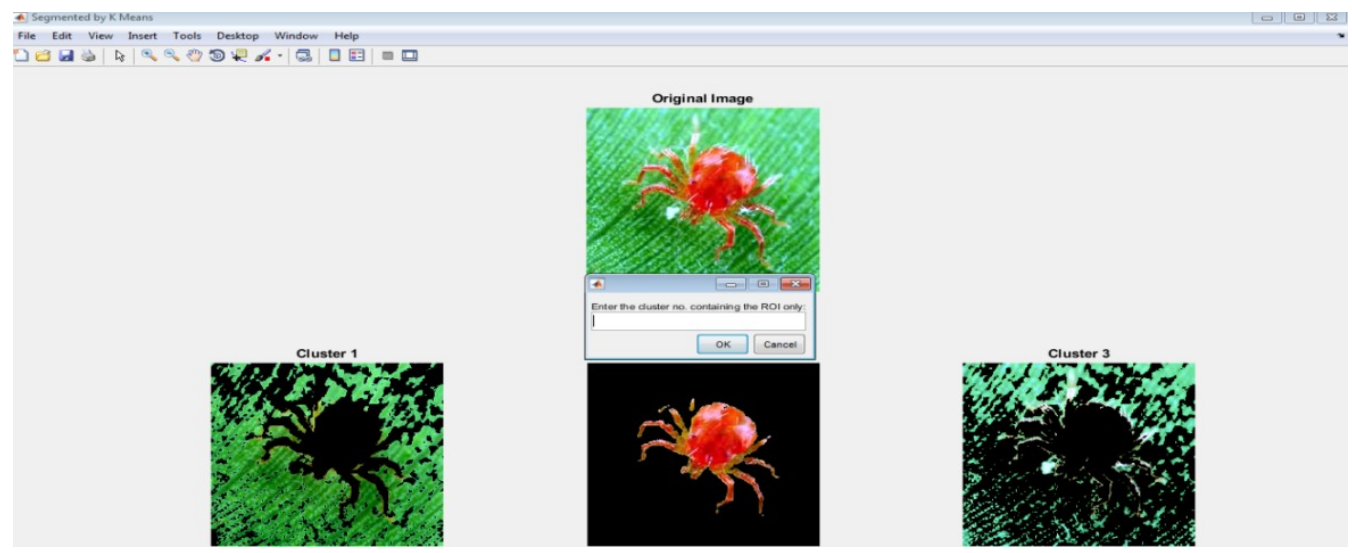

Fig 7: Image is divided in 3 clusters

The selected image is can be considered as the Segmented ROI (Region of Interest). Which is shown in the following snapshot :

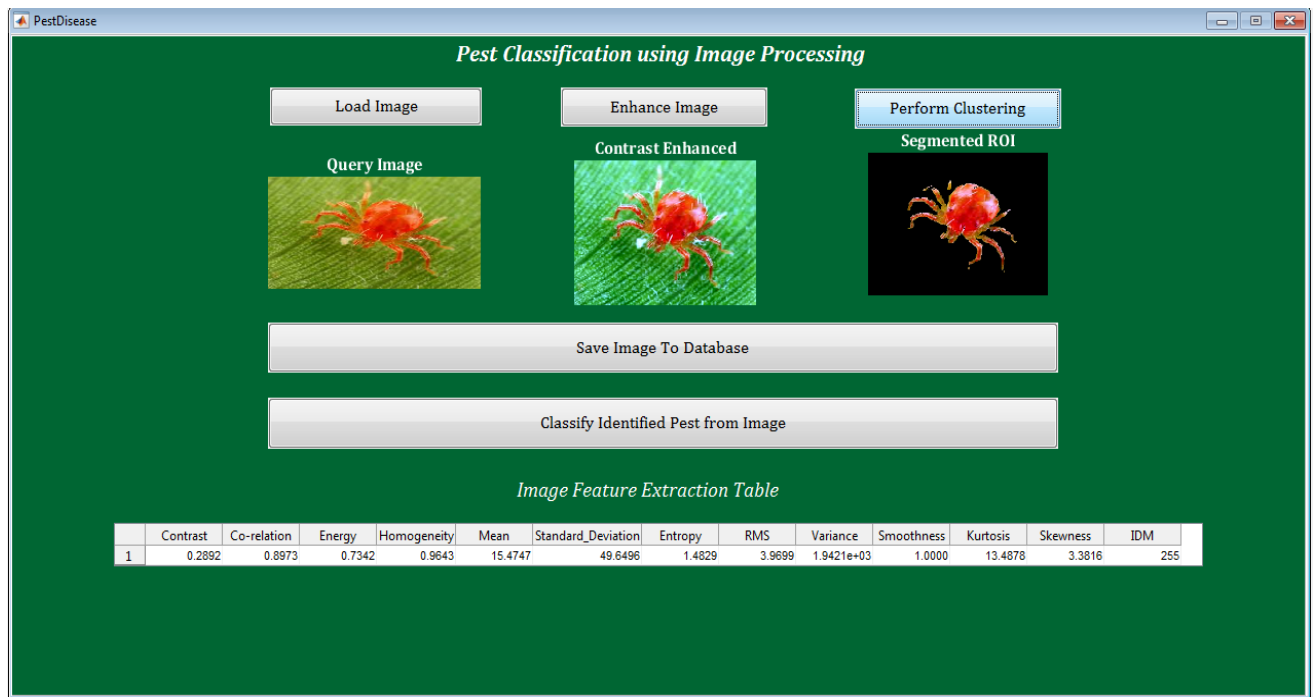

Fig 8: comparing image by using feature of images

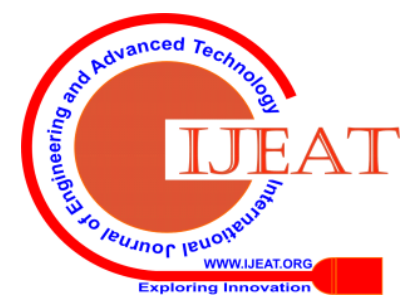




\section{Leaf Disease Classification using Advanced SVM Algorithm}

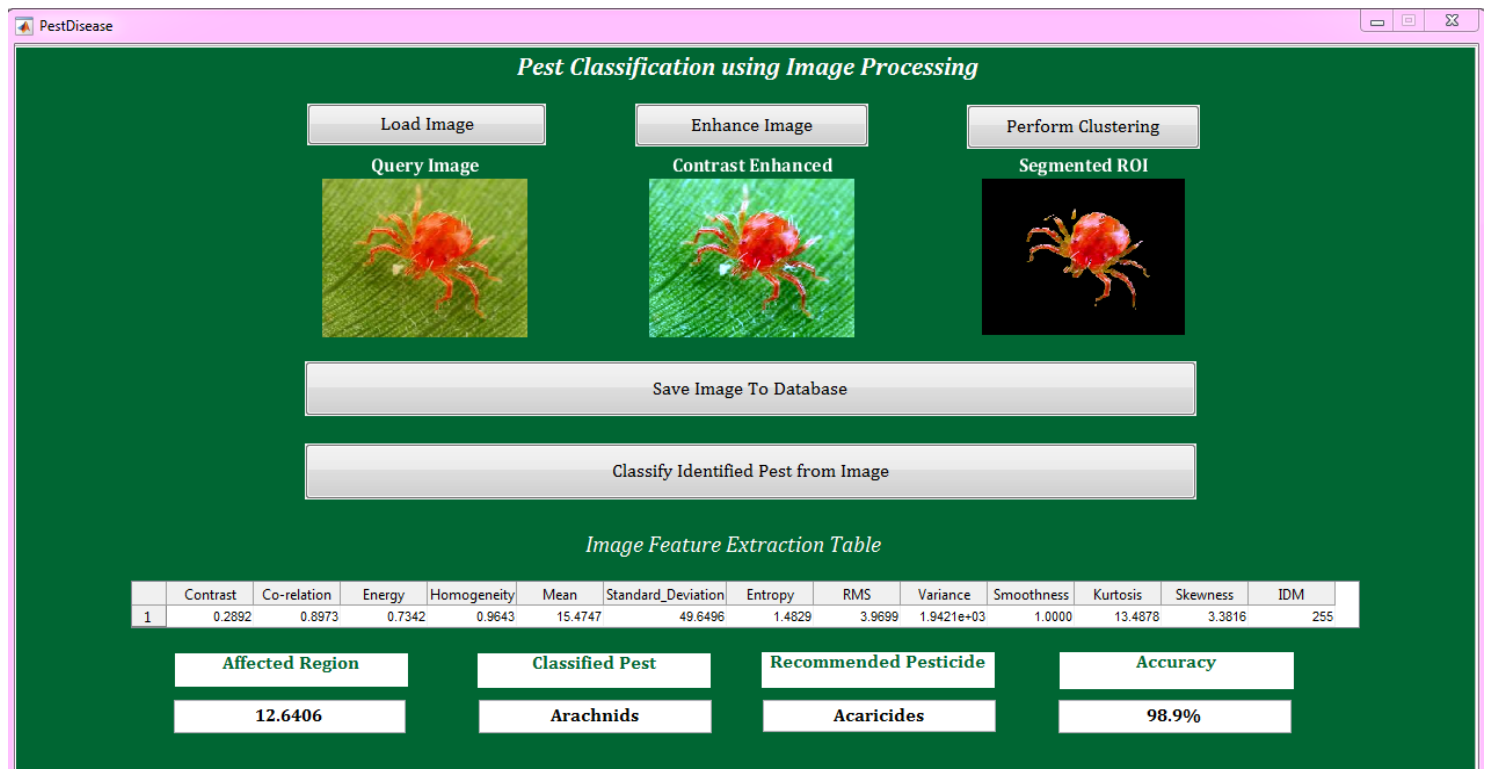

Fig 9: Extracting the features and suggesting pesticides

\section{RESUlt ANALYSIS}

Precision and recall are the two factors who plays very important role in such model evaluation metrics. Here precision can be defined as overall percentage of results and recall is the percentage of results produced by algorithm.

- Result (Accuracy) is measured by the 4 types of measures :

1) True positive (TP)

2) True Negative (TN)

3) False positive (FP)

4) False Negative (FN)

- $\quad$ Result $($ Acc. $)=\mathrm{TP}+\mathrm{TN} / \mathrm{TP}+\mathrm{TN}+\mathrm{FP}+\mathrm{FN}$

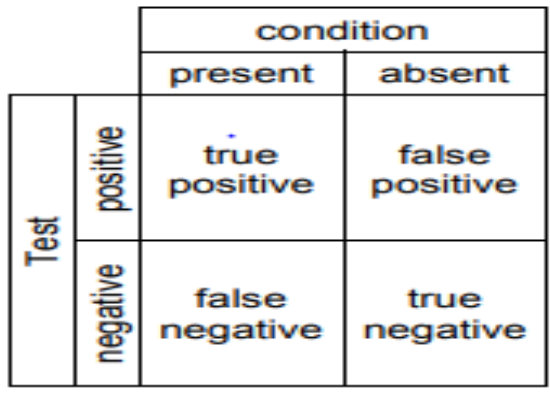

Table 10: Result chart

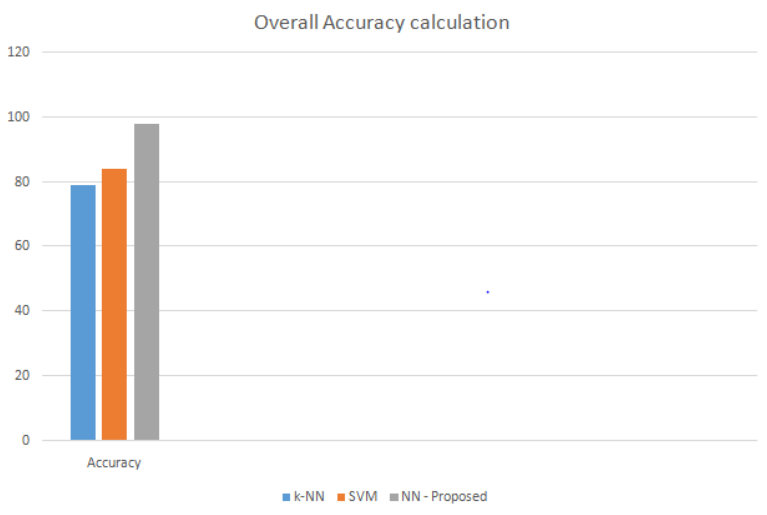

Fig 11 Overall Accuracy calculation

\section{CONCLUSION}

Legitimately there are various exchanges of pesticides and amazingly a noteworthy piece of the business is depends and using such dangerous substances to shields harvests to keep from bugs ambush and spreading of defilement. Such pesticides are really incredibly dangerous and used inorganic manufactured substances. Without a doubt, even some of such pesticides are profitable for bugs also. In reality, even a couple of times there is in like manner a believability that such synthetics may be subsequently washed during precipitation or watering the harvests. So the assessment since years on green house agro system base on early bug recognizable proof. Such system base on watching plants by camera. The photos gotten by cameras can be used to explored that atmosphere the plants are corrupted or not.

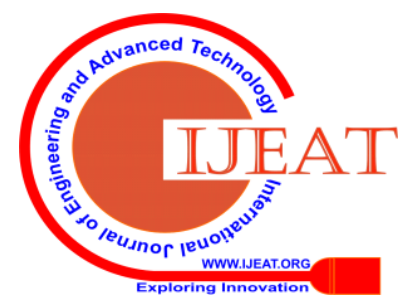


Different procedures and counts, for instance, concealing change, division, k-mean, knn, etc are used to requested such pictures. This investigation is focusing on the interpretation of picture for starting time trouble acknowledgment with the objective that the yield should be kept from mischief. The first objective is to detect some sample pest. To achieve this some digital image processing algorithms are acquired. The methods used are DBSCAN and $\mathrm{NN}$ algorithms. The overall early pest detection accuracy is $96 \%$ based on total pest dataset used in research.

\section{REFERENCES}

1. SantanuPhadikar and Jaya Sil "Rice Disease identification using Pattern Recognition Techniques" Proceedings of 11th International Conference on Computer and Information Technology (ICCIT 2008)2527 December, 2008, Khulna, Bangladesh, pp. 1-42442136$7 / 08$.

2. Dheeb Al Bashish, Malik Braik and SuliemanBaniAhmad "A Framework for Detection and Classification of Plant Leaf and Stem Diseases" 2010 IEEE International Conference on Signal and Image Processing, pp. 978-14244-8594-9/10.

3. Zulkifli Bin Husin, Abdul Hallis Bin Abdul Aziz, Ali Yeon Bin MdShakaff and RohaniBinti S Mohamed Farook "Feasibility Study on Plant Chili Disease Detection Using Image Processing Techniques" 2012 IEEE Third International Conference on Intelligent Systems Modelling and Simulation, pp. 978-0-7695-4668$1 / 12$.

4. Sabah Bashir and Navdeep Sharma "Remote Area Plant Disease Detection Using Image Processing" IOSR Journal of Electronics and Communication Engineering (IOSRJECE) ISSN : 2278-2834 Volume 2, Issue 6 (Sep-Oct 2012), PP 31-34.

5. Murali Krishnan and Dr.M.G.Sumithra "A Novel Algorithm for Detecting Bacterial Leaf Scorch (BLS) of Shade Trees Using Image Processing" 2013 IEEE 11th Malaysia International Conference on Communications 26th - 28th November 2013, Kuala Lumpur, Malaysia pp. 978-1-4799-1532-3/13

6. Ms. Kiran R. Gavhale, Prof. UjwallaGawande and Mr. Kamal O. Hajari "Unhealthy Region of Citrus Leaf Detection Using Image Processing Techniques" 2014 IEEE International Conference for Convergence of Technology, pp. 978-1-4799-3759-2/14.

7. Wan MohdFadzil W.M.N, Shah Rizam M.S.B and R. Jailani, Nooritawati M.T "Orchid Leaf Disease Detection using Border Segmentation Techniques" 2014 IEEE Conference on Systems, Process and Control (ICSPC 2014), 12 - 14 December 2014, Kuala Lumpur, Malaysia, pp. 978-1-4799-6106-1/14.

8. UsamaMokhtar, Mona A. S. Alit, Aboul Ella Hassenian, HeshamHefny "Tomato leaves diseases detection approach based on support vector machines" 2015 IEEE pp. 978-1-5090-0275-7/15.

9. D. A. Puspito Sari, I. Listiyowati, T. Nefianto, and Lasmono, "The Discrepancy between The Programs and Disaster Management Policy in Klapanunggal District, Bogor, West Java," IOP Conf. Ser. Earth Environ. Sci., vol. 135, no. 1, p. 012011, Mar. 2018. 